\title{
Medical futility treatment in intensive care units
}

\author{
Marko Jukić ${ }^{1}$, Lenko Šarić ${ }^{2}$, Ivana Prkić ${ }^{3}$, Livia Puljak ${ }^{4}$
}

${ }^{1}$ Department of Anesthesiology and Intensive Care, University Hospital Centre Split, Split, Croatia, ${ }^{2}$ Department of Anesthesiology and Intensive Care University Hospital Centre Split, Split Croatia, ${ }^{3}$ Department of Anesthesiology and Intensive Care, University Hospital Centre Split, Split, Croatia, ${ }^{4}$ Laboratory for Pain Research, University of Split School of Medicine, Split, Croatia

$$
\begin{aligned}
& \text { Correspondence: } \\
& \text { livia.puljak@mefst.hr } \\
& \text { Tel.: + } 38521557807 \\
& \text { Fax.: + } 38521557811
\end{aligned}
$$

Received: 4 November 2016

Accepted: 21 November 2016

Key words: Intensive care unit $\cdot$ Medical futility - End-of-life - Decision making Ethics.

\begin{abstract}
Objective. To investigate cases of potential medical futility treatment in intensive care unit (ICU). Materials and Methods. Retrospective review of 1567 charts of patients treated during the three-year period (2012 - 2014) in the ICU of the University Hospital Centre Split, Croatia, was conducted. More detailed analysis of the deceased patients' $(n=429)$ charts was performed to identify cases of potential medical futility treatment. There were 99 patients for which ICU treatment was questionable due to their low Glasgow coma scale (GCS) score. For those patients types and duration of treatment were analyzed. Results. Among patients who were treated during that period, $27 \%$ had died. Treatment of 99 patients (6.3\% of the deceased) was considered a potential medical futility. Mean age of those 99 patients was $68 \pm 14$ years and the mean stay in the ICU was $14 \pm 11$ days. They spent 1302 patient days in the ICU, of which $52 \%$ days they had GCS 3 score. They were treated with catecholamines during $40 \%$ of the patient days. Minimal therapy was provided during $44 \%$ of the patient days. Conclusions. Analysis of the deceased patients' charts in the ICU indicated that a certain percentage of patients did not need prolonged ICU treatment. Instead, they were supposed to be treated in a palliative care unit. To avoid medical futility treatment in ICUs, palliative care unit needs to be established, as well as protocols for determining medical futility cases and ethical committee that will decide which patients will be transferred to palliative care.
\end{abstract}

\section{Introduction}

Intensive care units (ICU) are units for treatment of patients with severely impaired organ function and life-threatening conditions. With expert and aggressive treatment, a patient's life may be saved, disability reduced and a patient may fully recover (1). There are different ICUs - general, surgical, infectious diseases, internal medicine, cardiac, gastrointestinal, pediatric, psychiatric, neurological, trauma, etc. If there is an adequate space, equipment and expert health workers, the same level of medical care can be provided on different clinics and depart- ments $(2,3)$. Due to rational care, high costs of treatment and lack of highly qualified medical personnel, smaller hospitals have central ICU where all patients who need mechanical ventilation, cardiovascular support and intensive care are treated (3). The aim of the ICU treatment is recovery of a failed organ function or of multiorgan failure, such as heart-, kidney- or liver failure, septic conditions, polytrauma, severe brain damage and metabolic disorders (4).

All treatments that physicians and other medical personnel provide to patients should primarily target prevention and maintenance of health, and when a patient 
presents with disease and injury, the main goal is to help the ill $(1,5)$. Emphasis should be on maintaining and, if possible, enhancing patients' quality of life and, at the same time, reducing physical and emotional pain and suffering $(5,6)$. Prolonging life in critically ill patients is not the goal in itself and it should not be the main goal of the treatment except when it is medically justified (7).

Palliative medicine is a new branch of medicine, caring for patients in end of life stage and patients for which medicine has exhausted all curative options and is unable to help a patient to restore health. Palliative care is an approach that helps patients faced with life-threatening illness and their families to improve their quality of life (8). This can be enabled via prevention and alleviation of symptoms using methods of early detection, assessment and pain management, and alleviating other physical, psychosocial and spiritual problems (8). Palliative care is necessary for chronic patients in acute progressive phases of disease, with life expectancy under 6 months, at a time when medical curative treatment is no longer effective (9). Considering ethical dilemmas faced by physicians daily, it is important to emphasize that physicians are not obliged to suggest or start treatment measures for which they believe will not improve patients' condition, but can prolong patients' suffering (4).

Dysthanasia is a concept opposite to the concept of "euthanasia", and it covers practice of extending biological life of a dying patient using medical treatments and technology without regard to the patient's quality of life. This term is now associated with medical futility. Although medical futility is a widely known concept, we still do not have a single definition of such procedures $(6,10,11)$. It has been reported previously that different institutions have different approaches to what is considered a medical futility treatment (2).

The factors associated with differences in the utilization of ICU resources between institutions are the decision-making process about when to start and complete treatment, the patient's condition at the moment of ICU admission, the number of staff employed and the possibility of transferring patients to other organizational units after completed treatment (2). Existing evidence indicates that providing proactive palliative care in the ICU, using either consultative or integrative palliative care interventions, may decrease hospital and ICU length of stay. Furthermore, it does not affect satisfaction, and neither decreases or affects patients' mortality (5). Therefore, it is important to continue discussion about medical futility treatment in order to prevent practice of dysthanasia and to provide patients with the best care without unnecessarily prolonging their life.

The aim of the study was to explore potential cases of medical futility treatment in the ICU and to encourage establishment of palliative care unit in the hospital, algorithms for recognizing patients that may be subjected to medical futility and working protocols for the ICU. The goal was also to point out the necessity of forming ethical committee that could be involved in decision-making, regarding potential medical futility and provide recommendations or decisions about further proceedings.

\section{Materials and methods}

\section{Patients}

Retrospective review of 1567 charts of patients treated during the three year period (2012-2014) in ICU of the University Hospital Split, Croatia, was conducted.

\section{Setting}

This is a central-type ICU in a tertiary hospital, organized within the Department of anesthesiology and intensive care. The ICU 
operates on two locations, one with 10 and another one with 8 beds. The hospital has a total of 1530 beds, with 23 beds for intensive treatment (18 beds in the general ICU and 5 beds for the postoperative cardiosurgical ICU). Heads of the ICUs are anesthesiologists with a subspecialty in intensive treatment.

\section{Data analysis}

Patient records and daily charts of deceased patients $(429 / 1567 ; 27 \%)$ were analyzed in detail. The following data were recorded: diagnosis, therapy and procedures received in the ICU (resuscitation, type of pharmacological treatment, minimal therapy), duration of treatment in the ICU before death, Glasgow coma scale (GCS) score for assessment of impairment of consciousness in response to defined stimuli, disease severity according to the Acute Physiology and Chronic Health Evaluation II (APACHE II) classification and hospital mortality estimate using Simplified Acute Physiology Score II (SAPS II) criteria. Additionally, bed occupancy in the ICU during a year was analyzed and indicated in percentages. Deceased patients with potential medical futility treatment were defined using the GCS (GCS $<8$ during ICU stay) and using those criteria 99 patients $(6.3 \%$ of the total number of treated patients) were identified.

For evaluating whether their treatment in the ICU was justified, those 99 patients were divided into the 4 groups: the first group were the patients with GCS 3 who received minimal therapy in the ICU (5\% glucose or saline and mechanical ventilation); the second group were those with GCS $<6$, who received vasopressor support with catecholamines (epinephrine, norepinephrine, dobutamine, dopamine); in the third group patients had GCS $<6$ and did not receive catecholamine treatment, while in the fourth group patients had GCS above 6 and received support with catecholamines (norepinephrine, dobutamine, dopamine). Data were shown separately for two different ICU locations. Economical aspects of treatment were not analyzed considering inadequate reimbursement system mandated by the Croatian Health Insurance Fund, which is not commensurate with the actual expenses.

\section{Ethics}

The study was approved by the University Hospital Split Ethical Committee. The study was conducted in accordance with the Declaration of Helsinki from 1975 and its amendments from 1983.

\section{Statistics}

For testing normality of data we used Kolmogorov-Smirnov test. Data were analyzed using descriptive statistics and shown as frequencies, percentages, means, standard deviation, median and range. For data analysis GraphPad program was used (GraphPad Software, La Jolla, CA, USA).

\section{Results}

In the two ICUs of the University Hospital Split, 1567 patients were treated during the analyzed period of three years (2012-2014), of which 429 patients died (27\%). Detailed analysis was conducted for 99 patients (6.3\%) that were potential cases of medical futility treatment (59 patients in Unit I and 40 patients in Unit II). Among them, there were 66 men and 33 women. Considering the four different groups in which they were categorized, according to their condition, most of the patients were in the group 2 (Table 1).

Patient characteristics are shown in Table 2. Mean age of patients with potential medical futility treatment was $68 \pm 14$ years and their mean stay in the ICU was $14 \pm 11$ 
Table 1 Number of patients in ICU ${ }^{*}$ in different categories of potential medical futility $(n=99)$

\begin{tabular}{lllll}
\hline Groups & GCS $^{+}$ & $\begin{array}{l}\text { Vasopressor } \\
\text { support }\end{array}$ & $\begin{array}{l}\text { Number of } \\
\text { patients in } \\
\text { both units }\end{array}$ & $\mathrm{n}(\%)$ \\
\hline First & 3 & Yes $^{\ddagger}$ & 9 & $9(9)$ \\
Second & $<6$ & Yes & 62 & $62(63)$ \\
Third & $<6$ & No & 15 & $15(15)$ \\
Fourth & $<8$ & Yes & 13 & $13(13)$ \\
\hline
\end{tabular}

"Intensive care unit; ${ }^{\dagger}$ Glasgow Coma Scale; ${ }^{\ddagger}$ Initially afterwards minimal therapy.

days. Duration of patients' stay in the ICU is shown in Table 2 for each group, as well as their APACHE II and SAPS II scores.

Total number of days patients were treated in the ICU is shown in Table 3. Patients spent 1302 patient days ( 1 to 79 ) in the ICU. GCS score of 3 was observed during 675 (52\%) patient days of their ICU stay, and catecholamines were administered to those patients for $520(40 \%)$ days. Nine patients received minimal therapy for a total of 55 days ( $4 \%$ of the total days that those patients spent in the ICU).

In 9 patients who received minimal therapy during the ICU stay, that therapy was administered during $33 \%$ of patient days spent in the ICU. They had GCS 3 during $65 \%$ of the patient days (Table 4 ).

Bed occupancy in the ICU during the year 2012 is shown in Table 5. Beds in the Unit I were occupied from 80 to $100 \%$ during 198 days in the year (54\%), while the bed

Table 2 Characteristics of patients in ICU ${ }^{*}$ with potential medical futility treatment $(n=99)$

\begin{tabular}{lllll}
\hline \multirow{2}{*}{ Variables } & Group 1 & Group 2 & Group 3 & Group 4 \\
\cline { 2 - 4 } & $\begin{array}{l}\mathrm{GCS}^{\dagger}=3 \\
\text { min. th. }\end{array}$ & $\begin{array}{l}\text { GCS } \\
<6+\mathrm{VP}^{\S}\end{array}$ & $\begin{array}{l}\text { GCS } \\
<6\end{array}$ & $\begin{array}{l}\mathrm{GCS}^{\dagger} \\
>6+\mathrm{VP}^{\S}\end{array}$ \\
\hline Number of patients & 9 & 62 & 15 & 13 \\
Median age in years (range) & $62(35-84)$ & $66.5(22-84)$ & $73(52-82)$ & $73(24-86)$ \\
$\begin{array}{l}\text { Median stay in } \\
\text { ICU* (days; range) }\end{array}$ & $16(4-48)$ & $9.5(1-79)$ & $7(3-51)$ & $9(4-33)$ \\
APACHE II\| & 24.5 & 24.2 & 24.6 & 23 \\
SAPS IIף & 67.1 & 68.3 & 72.3 & 63.5 \\
\hline
\end{tabular}

"Intensive care unit; ${ }^{\dagger}$ Glasgow Coma Scale; ${ }^{\ddagger}$ Minimal therapy; ${ }^{5}$ Vasopressor support "Acute Physiology and Chronic Health Evaluation II, I"Simplified Acute Physiology Score II.

Table 3 Number of patient days in ICU* and therapy $(\mathrm{n}=99)$

\begin{tabular}{lllll}
\hline \multirow{2}{*}{ Groups } & Days in the ICU* & $\begin{array}{l}\text { Days with } \\
\mathrm{GCS}^{\dagger}=3\end{array}$ & $\begin{array}{l}\text { Days receiving } \\
\text { VP\$ }\end{array}$ & $\begin{array}{l}\text { Days receiving } \\
\text { min. th. }\end{array}$ \\
\cline { 2 - 5 } & $\mathrm{n}$ & $\mathrm{n}(\%)$ & $\mathrm{n}(\%)$ & $\mathrm{n}(\%)$ \\
\hline $\begin{array}{l}\text { First } \\
\text { (Minimal therapy) }\end{array}$ & 166 & $108(65)$ & $55(33)$ & $55(33)$ \\
$\begin{array}{l}\text { Second } \\
\left(\mathrm{GCS}^{\dagger}<6+\mathrm{VP}^{\S}\right)\end{array}$ & 794 & $445(56)$ & $338(43)$ & 0 \\
$\begin{array}{l}\text { Third } \\
\left(\mathrm{GCS}^{\dagger}<6\right)\end{array}$ & 168 & $122(73)$ & 0 & 0 \\
$\begin{array}{l}\text { Fourth } \\
(\mathrm{GCS}\end{array}$ & 174 & 0 & $127(73)$ & 0 \\
\hline Total & 1302 & $675(52)$ & $520(40)$ & $55(4)$ \\
\hline
\end{tabular}

"Intensive care unit; ${ }^{\star}$ Glasgow Coma Scale; ${ }^{5}$ Vasopressor support; ${ }^{\ddagger}$ Minimal therapy. 
Table 4 Patients receiving minimal therapy during their ICU* stay

\begin{tabular}{llllll}
\hline Patient number & Age & $\begin{array}{l}\text { Days of } \\
\text { ICU* stay }\end{array}$ & $\begin{array}{l}\text { Days of } \\
\mathrm{GCS}^{+}=3\end{array}$ & $\begin{array}{l}\text { Days of } \\
\text { VPs }\end{array}$ & $\begin{array}{l}\text { Days of } \\
\text { min. th. }\end{array}$ \\
\hline 1 & 35 & 16 & 16 & 0 & 10 \\
2 & 55 & 21 & 16 & 4 & 5 \\
3 & 62 & 4 & 1 & 2 & 5 \\
4 & 82 & 8 & 8 & 20 & 13 \\
5 & 84 & 23 & 16 & 17 & 6 \\
6 & 71 & 7 & 6 & 0 & 7 \\
7 & 56 & 13 & 7 & 9 & 3 \\
8 & 66 & 26 & 9 & 4 & 4 \\
\hline 9 & 40 & $166(100 \%)$ & $108(65 \%)$ & $55(33 \%)$ & $55(33 \%)$ \\
\hline Total & & & & & \\
\hline
\end{tabular}

"Intensive care unit; ${ }^{\dagger}$ Glasgow Coma Scale; ${ }^{\S}$ Vasopressor support; ${ }^{\ddagger}$ Minimal therapy

occupancy in Unit II was above 80\% during $119(33 \%)$ of days during that year. Similar data were observed for years 2013 and 2014.

Among 99 selected patients, $75 \%$ of them stayed for up to 9 days in the ICU, while $16 \%$ of patients in UNIT I and $11 \%$ of patients in UNIT II stayed for 10 to 19 days in the ICU. There was $1 \%$ of patients who were treated in the ICU longer than 20 days (Table 6).

Table 5 Bed occupancy in the Unit I and Unit II during year 2012

\begin{tabular}{lll}
\hline Unit I (10 beds) & & \\
\hline Beds & Days & $\%$ \\
\hline 10 & 45 & 100 \\
9 & 73 & 90 \\
8 & 80 & 80 \\
7 & 62 & 70 \\
6 & 50 & 60 \\
5 & 31 & 50 \\
\hline Unit II (7 beds + one bed for burns) & \\
\hline Beds & Days & $\%$ \\
\hline $7+1$ & 4 & 100 \\
7 & 41 & 100 \\
6 & 74 & 85.7 \\
5 & 89 & 71.4 \\
4 & 87 & 57.1 \\
3 & 43 & 42.8 \\
2 & 28 & 28.5 \\
\hline
\end{tabular}

Table 6 Duration of patient stay in the Unit I and Unit II during year 2012

\begin{tabular}{lll}
\hline \multirow{2}{*}{ Number of days in the ICU* } & Unit I & Unit II \\
\cline { 2 - 3 } & $\mathrm{n}(\%)$ & $\mathrm{n}(\%)$ \\
\hline 1 to 9 & $262(74)$ & $128(76)$ \\
10 to 19 & $58(16)$ & $19(11)$ \\
20 to 29 & $21(5.9)$ & $8(4.7)$ \\
30 to 39 & $4(1.2)$ & $5(3.0)$ \\
40 to 49 & $6(1.7)$ & $3(1.8)$ \\
50,52 & $1(0.3)$ & $1(0.6)$ \\
75,76 & $1(0.3)$ & $2(1.2)$ \\
85,86 & $0(0)$ & $2(1.2)$ \\
141 & $0(0)$ & $1(0.6)$ \\
\hline Total & $353(100)$ & $169(100)$ \\
\hline
\end{tabular}

"Intensive care unit.

Details about 6 patients with GCS 3 who were resuscitated and received catecholamines are shown in Table 7. Due to GCS 3 results, these patients were resuscitated without expecting recovery. On average, they had GCS 3 during $56 \%$ of patient days spent in the ICU. During $56 \%$ of days spent in the ICU, they received catecholamine support.

The most common diagnoses of the 99 patients for which it was estimated that they received medical futility treatment were respiratory failure, injuries - most commonly 
Table 7 Patients that were resuscitated without expecting recovery (6 patients)

\begin{tabular}{lllll}
\hline Patient number & $\begin{array}{l}\text { Days } \\
\text { in ICU }\end{array}$ & $\begin{array}{l}\text { Days with } \\
\text { GCS 3 }\end{array}$ & $\begin{array}{l}\text { Days with } \\
\text { vasopressor support }\end{array}$ & $\begin{array}{l}\text { Times } \\
\text { resuscitated (n) }\end{array}$ \\
\hline 1 & 24 & 10 & 18 & 3 \\
2 & 5 & 3 & 0 & 2 \\
3 & 6 & 2 & 4 & 1 \\
4 & 5 & 5 & 4 & 1 \\
5 & 4 & 2 & 2 & 1 \\
6 & 6 & 6 & 0 & 1 \\
\hline Total & $50(100 \%)$ & $28(56 \%)$ & $28(56 \%)$ & - \\
\hline
\end{tabular}

Table 8 Patients' diagnoses $(\mathrm{n}=99)$

\begin{tabular}{ll}
\hline Main diagnosis & $\mathrm{n}$ \\
\hline J96 (Respiratory failure) & 20 \\
S02, S06 and S09 (Head injuries) & 19 \\
I61 (Intracerebral haemorrhage) & 13 \\
I21, I60, 162, I63 and I71 (Acute myocardial infarction and Cerebrovascular diseases) & 11 \\
I46 (Cardiac arrest) & 8 \\
A41,9 (Sepsis) & 8 \\
C15, C16 - C20, C34, C71 and C93 (Malignant neoplasms of digestive organs, bronchus & 8 \\
and lungs, brain and laeukemias) & 3 \\
I26 and I72 (Pulmonary embolism and aneurysm) & 2 \\
J80 and J95 (ARDS* and postprocedural respiratory disorders) & 2 \\
X61 (Intentional self-poisoning) & 2 \\
G06 and G37,3 (Intracranial abscess and granuloma and acute transverse myelitis) & 1 \\
A39,9 (Meningococcal infection) & 1 \\
K56 (Paralytic ileus and intestinal obstruction) & 1 \\
T31,5 (Burns) & 2 \\
\hline
\end{tabular}

"Adult respiratory distress syndrome; Codes for diagnoses shown according to the International Classification of Diseases (ICD-10). Available at: http://apps.who.int/classifications/icd10/browse/2010/en.

from traffic accidents, circulation failure, sepsis and postoperative care of patients suffering from malignant diseases (Table 8).

\section{Discussion}

We conducted a retrospective chart review to investigate cases of potential medical futility treatment among deceased patients in intensive care unit. Our analysis indicated that a certain percentage of patients did not need prolonged ICU treatment. Instead, they were supposed to be treated in a palliative care unit.

Technology has revolutionized all aspects of our lives, and medicine is not an exception. Diagnostic procedures and interventions that can be done today were once unthinkable. By using technology and new knowledge from research, intensive care of patients is now on a very high level (10). However, technological advancement has brought along a number of questions in the field of medical ethics $(4,12)$. Due to 
the complexity of decision-making in lifethreatening conditions, ethical committees are being established. Their purpose is to provide help in solving ethically complex situations and to give recommendations about managing certain cases (11). Based on the objective medical factors, a good assessment of treatment outcomes can be made and further medical procedures directed (13). Likewise, decision-making about further treatment should always take into account patients' quality of life (10). When everything possible has been done, and when there is no chance of patient's recovery and further treatment is useless, certain complex medical interventions should no longer be conducted $(12,14)$. A patient without chances of recovery will be medically treated, but extraordinary treatment measures should not be taken in the case of organ failure (1). Certain institutions have protocols for terminating treatment in specific situations, when patient is disconnected from the mechanical ventilation and resuscitation procedures are not conducted. Such protocols can be devised in the hospital ICUs where working protocols are adopted, where ethical committees give consent for ending further intensive treatment because it is futile, and when there are no objective chances that patient will recover in a way he/she will have an acceptable quality of life $(15,16)$.

In our analysis of one ICU over three years (2012-2014), based on the criteria set for medical utility, $6.3 \%$ of deceased patients received treatment which was probably medical futility. That number is probably even greater, considering that data from literature describe that physicians consider $8.6 \%$ of ICU patients receive potentially futile treatment, and $11 \%$ definitely receive futile treatment (7). Average age of our patients considered potential medical futility was 68 years. They spent a total of 1302 days in the ICU, 14 days on average. According to the ETHICUS study, average ICU stay of pa- tients considered potential medical futility was 18 days, and 15 days for patients considered definitive medical futility (7). Among patients considered as potential medical futility, $87 \%$ had GCS score under 6 , and some of them had that score on the admission to the ICU. Patients had GCS 3 during 675 patient days (52\% of their ICU stay). Only 9\% of patients received minimal therapy. Catecholamine support was given to $76 \%$ of the potential medical futility patients during $40 \%$ of their ICU patient days. Considering that their need for further intensive care was questionable, it is unclear why potential medical futility patients received catecholamine support $(7,17)$.

In the analyzed ICU, 9 patients that were considered potential medical futility received minimal therapy, which means that their active treatment was withdrawn during their ICU stay ( $33 \%$ of their ICU stay). Those patients had GCS 3 during $65 \%$ of the ICU time. These data are in accordance with the corresponding data in other settings. Study from UK reported that active treatment was withdrawn for $9 \%$ of patients admitted to ICU (12). Time between ICU admission and decision about ending active treatment should be as short as possible. A large study of more than 80,000 ICU admissions in England and Wales showed that if that time could be shortened for just one day, it was estimated that 100 patients could be saved in that region annually $(12,18)$.

Capacities of ICU units are limited and treating patients whose recovery is not expected is not justified. Bed occupancy in the two analyzed ICU units were over $80 \%$ during $54 \%$ of the year for UNIT I and during $33 \%$ of the year for UNIT II, respectively. That should never be above $85 \%$ to allow admission of acute patients. Therefore, it is important to establish criteria for ICU admission. If the ICU receives and treats patients that cannot profit from that care, this practice reduces available beds and resources for 
treating patients that really need intensive treatment (19). In adition to clear admission criteria, protocols for determining medical futility treatment should also be established as well as palliative care unit $(11,19)$.

Our data indicate that $25 \%$ of patients considered as potential cases of medical futility treatment were treated in the ICU longer than 10 days; $11 \%$ of those patients spent more than 20 days in the ICU. On average, those patients spent 13 days in the ICU. For comparison, average duration of ICU stay in Finland is 4 days and in Poland 14 days (2).

Data from the United States of America indicate that length of stay increases costs of intensive treatment, while not necessarily prolonging survival. Stricker et al. showed that caring for $11 \%$ of patients who were treated in the ICU longer than 7 days accounts for $50 \%$ of ICU costs (20). Several studies indicated a $50 \%$ mortality of patients who stay in ICU longer than 14 days, while $70 \%$ of those patients achieve less than $50 \%$ of functional recovery $(21,22)$. These data are additional support for arguments that medical futility treatments in the ICU should be reduced to minimum. Patients with GCS 3 that were resuscitated and received catecholamines on average had GCS 3 during $56 \%$ of days during their ICU stay and $56 \%$ of days received catecholamines. Since recovery objectively was not expected, it is reasonable to question decision to attempt resuscitation, especially considering that some patients were resuscitated multiple times. Furthermore, it is unclear why patients with no chances of improvement receive catecholamine support. There is no professional, ethical or economical justification for attempting resuscitation and giving catecholamines to patients without chances of recovery $(17,23)$.

Various reasons may explain why those patients were resuscitated. Firstly, it could be due to insufficient communication among staff and between patients and patients' family. Secondly, a physician might feel that his or her duty is to do "everything for a patient". One of the reasons is probably insufficient knowledge of patients' wishes, i.e. lack of official forms for resuscitation that patients can sign and his family can use when a patient is no longer capable of making decisions $(23,24)$.

One of the reasons that may explain such prevalence of medical futility is lack of palliative care unit in the analyzed hospital. Such units could receive medical futility patients once their intensive treatment is no longer considered sensible (10). The second reason is the lack of working protocols, algorithms and guidelines that will help physicians to decide that further treatment is futile (2). Furthermore, in the analyzed hospital there is no ethical committee in charge of making decisions about medical futility and helping physicians with decision-making about potential medical futility cases. Introduction of such guidelines and an ethical committee would help physicians and nurses in their daily work. Until such instruments become available, health care workers are forced to provide advanced treatments to ICU patients, knowing that they are futile $(10,11,14)$. This practice prolongs suffering of patients and their family, and it also contributes to increased cost of health care. Physicians are burdened with conflict between their understanding of situation and requirements for treatments and they are growing dissatisfied. Futile medical care is provided because of fear of legal consequences, limited and unsatisfactory communication between physicians, patients and their family, and insufficient knowledge of palliative care principles $(10,14)$. Tools such as SAPS II and APACHE II have an excellent ability to discriminate between survivors and non-survivors so they can be used to guide medical decisions (25).

Death is natural and final part of life. Medicine can not prolong that process in- 
definitely. When it is clear that inevitable death is imminent, it is legitimate for a physician to refuse or limit treatment options that would only secure a precarious and burdensome prolongation of life, for as long as basic humane, compassionate care is not interrupted (14). It is also accepted and ethically justified to refuse forms of treatment that are useless and not beneficial to a patient or in situations when it is not possible to offer such treatment (11).

Intensive treatment is very expensive and should be used rationally. Cost of treatment was not calculated in the study because calculation of medical expenses in Croatia is very specific and determined by the Croatian Health Insurance Fund, and therefore it cannot be compared to costs of treatment in other high-income countries. However, based on the published data, costs of ICU treatment account for $15 \%-20 \%$ of hospital expenses, i.e. more than one third of expenses of the entire health system (15). After taking into account that a part of those funds was spent on medical futility treatments, on patients that should not be treated in the ICU at all, it is clear that this is a significant financial consideration. By treating in the ICU patients that cannot benefit from that treatment, other patients who may benefit from intensive care may be refused such treatment (1). Considering that ICUs are usually of limited capacities and often fully booked, from a medical, ethical and economical point of view it is not justified to use it for medical futility treatments (1).

All patients analyzed in the study as potential medical futility treatment cases died in the ICU. Their transfer to palliative care unit was not possible because there was no such unit, not in that hospital nor anywhere nearby in that geographical region. Likewise, there were no protocols to conduct in situations when ICU treatment was useless, or protocols for detaching a patient from mechanical ventilation when such treatment was no longer sensible.

\section{Conclusions}

Analysis of patient charts of patients who died in the ICU indicates that around 6\% of patients were not supposed to be treated in the ICU - those patients belonged to the palliative care unit. Since this hospital does not have palliative care unit, nor such unit exists nearby, it is necessary to establish such unit for appropriate patient care. Besides establishing palliative care unit and a team, the hospital also needs to establish ethical committee that will consider all cases of potential medical futility treatment. Furthermore, clear working protocols for ICU are necessary. Such guidelines would help patients make decisions about resuscitation and providing vasoactive support. Additionally, physicians should receive education about medical futility.

\footnotetext{
What is already known on this topic

Emphasis of a medical treatment in intensive care units should be on maintaining and, if possible, enhancing patients' quality of life and, at the same time, reducing physical and emotional pain and suffering. Prolonging life in critically ill patients is not the goal in itself and it should not be the main goal of treatment except when it was medically justified.
}

What this study adds

A certain percentage of deceased patients did not need prolonged treatment in intensive care unit. Such patients should be treated in a palliative care unit instead. To avoid medical futility treatment, palliative care unit needs to be established, as well as protocols for determining medical futility cases and ethical committee that will decide which patients will be transferred to the palliative care.

Authors' contributions: Conception and design: MJ; Acquisition, analysis and interpretation of data: MJ, LS , IP and LP. Drafting the article: MJ, LŠ, IP and LP; Revising it critically for important intellectual content: MJ, LŠ, IP and LP; Approved final version of the manuscript: MJ, LŠ, IP and LP.

Conflict of interest: The authors declare that they have no conflict of interest. 


\section{References}

1. Orsini J, Butala A, Ahmad N, Llosa A, Prajapati R, Fishkin E. Factors influencing triage decisions in patients referred for ICU admission. J Clin Med Res. 2013;5(5):343-9.

2. Adamski J, Goraj R, Onichimowski D, Gawlikowska E, Weigl W. The differences between two selected intensive care units located in central and northern Europe - preliminary observation. Anaesthesiol Intensive Ther. 2015;47(2):117-24.

3. Haupt MT, Bekes CE, Brilli RJ, Carl LC, Gray AW, Jastremski MS, et al. Guidelines on critical care services and personnel: Recommendations based on a system of categorization of three levels of care. Crit Care Med. 2003;31(11):2677-83.

4. Truog RD, Campbell ML, Curtis JR, Haas CE, Luce JM, Rubenfeld GD, et al. Recommendations for end-of-life care in the intensive care unit: a consensus statement by the American College [corrected] of Critical Care Medicine. Crit Care Med. 2008;36(3):953-63.

5. Aslakson R, Cheng J, Vollenweider D, Galusca D, Smith TJ, Pronovost PJ. Evidence-based palliative care in the intensive care unit: a systematic review of interventions. J Palliat Med. 2014;17(2):219-35.

6. Schneiderman LJ, Faber-Langendoen K, Jecker NS. Beyond futility to an ethic of care. Am J Med. 1994;96(2):110-4.

7. Huynh TN, Kleerup EC, Wiley JF, Savitsky TD, Guse D, Garber BJ, et al. The frequency and cost of treatment perceived to be futile in critical care. JAMA Intern Med. 2013;173(20):1887-94.

8. World Health Organization [homepage on the Internet]. Definition of Palliative Care [Updated 2016, cited 2016 Nov 22]. Available from: http:// www.who.int/cancer/palliative/definition/en/.

9. Campbell ML, Guzman JA. Impact of a proactive approach to improve end-of-life care in a medical ICU. Chest. 2003;123(1):266-71.

10. Gristina GR, De Gaudio R, Mazzon D, Curtis JR. End of life care in Italian intensive care units: where are we now? Minerva Anestesiol. 2011;77(9):911-20.

11. Wilkinson DJ, Savulescu J. Knowing when to stop: futility in the ICU. Curr Opin Anaesthesiol. 2011;24(2):160-5.

12. Wilkinson DJC SJ. A costly separation between withdrawing and withholding treatment in intensive care. Bioethics. 2014;28(3):127-37.

13. Fukuhara T, Aoi M, Namba Y. Mechanical ventilation for comatose patients with inoperative acute intracerebral hemorrhage: possible futility of treatment. PLoS One. 2014;9(7):e103531.
14. Manalo MF. End-of-Life Decisions about Withholding or Withdrawing Therapy: Medical, Ethical, and Religio-Cultural Considerations. Palliat Care. 2013;7:1-5.

15. Gruenberg DA, Shelton W, Rose SL, Rutter AE, Socaris S, McGee G. Factors influencing length of stay in the intensive care unit. Am J Crit Care. 2006;15(5):502-9.

16. Dowdy MD, Robertson C, Bander JA. A study of proactive ethics consultation for critically and terminally ill patients with extended lengths of stay. Crit Care Med. 1998;26(2):252-9.

17. Masood UR, Said A, Faris C, Al Mussady M, Al Jundi A. Limiting intensive care therapy in dying critically Ill patients: Experience from a tertiary care center in United Arab Emirates. Int J Crit Illn Inj Sci. 2013;3(3):200-5.

18. Bertolini G, Boffelli S, Malacarne P, Peta M, Marchesi M, Barbisan C, et al. End-of-life decisionmaking and quality of ICU performance: an observational study in 84 Italian units. Intensive Care Med. 2010;36(9):1495-504.

19. Orsini J, Blaak C, Yeh A, Fonseca X, Helm T, Butala A, et al. Triage of Patients Consulted for ICU Admission During Times of ICU-Bed Shortage. J Clin Med Res. 2014;6(6):463-8.

20. Stricker K, Rothen HU, Takala J. Resource use in the ICU: short- vs. long-term patients. Acta Anaesthesiol Scand. 2003;47(5):508-15.

21. Ryan TA, Rady MY, Bashour CA, Leventhal M, Lytle B, Starr NJ. Predictors of outcome in cardiac surgical patients with prolonged intensive care stay. Chest. 1997;112(4):1035-42.

22. Fakhry SM, Kercher KW, Rutledge R. Survival, quality of life, and charges in critically III surgical patients requiring prolonged ICU stays. J Trauma. 1996;41(6):999-1007.

23. Cohn S, Fritz ZB, Frankau JM, Laroche CM, Fuld JP. Do Not Attempt Cardiopulmonary Resuscitation orders in acute medical settings: a qualitative study. QJM. 2013;106(2):165-77.

24. Fritz Z, Malyon A, Frankau JM, Parker RA, Cohn $\mathrm{S}$, Laroche CM, et al. The Universal Form of Treatment Options (UFTO) as an alternative to Do Not Attempt Cardiopulmonary Resuscitation (DNACPR) orders: a mixed methods evaluation of the effects on clinical practice and patient care. PLoS One. 2013;8(9):e70977.

25. Godinjak A, Iglica A, Rama A, Tancica I, Jusufovic S, Ajanovic A, et al. Predictive value of SAPS II and APACHE II scoring systems for patient outcome in a medical intensive care unit. Acta Med Acad. 2016;45(2):97-103. 